\title{
Breastfeeding Rates Among Singletons, Twins and Triplets in Japan: A Population-Based Study
}

\author{
Yoshie Yokoyama,' Saeko Wada, ${ }^{2}$ Masako Sugimoto, ${ }^{2}$ Mari Katayama, ${ }^{2}$ Miyuki Saito, ${ }^{2}$ and Jun Sono ${ }^{2}$ \\ ' Faculty of Health Sciences, Okayama University Medical School, Okayama City, Japan \\ ${ }^{2}$ Nishinomiya City Public Health Center, Nishinomiya City, Japan
}

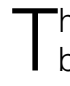
his study was performed to determine the rates of breastfeeding among singletons, twins and triplets in Japan, and identify factors associated with the decision to breastfeed or bottle-feed. We analyzed a database of medical check-up of infants aged 3 to 6 months between April 2001 and July 2004 in Nishinomiya City in Japan. This medical check-up is given to almost $100 \%$ of infants in Japan and the data of 15,262 infants were analyzed. Among these, 14,963 (98.0\%) were singletons, 290 (1.9\%) were twins and $9(0.1 \%)$ were triplets. Exclusive breastfeeding was chosen by 6680 (43.8\%) mothers, mixed-feeding by 4645 (30.4\%) mothers, and bottlefeeding with formula milk only by 3900 (25.6\%) mothers. The rate of exclusive breastfeeding among twins or triplets was significantly lower than among singleton babies: $4.1 \%$ among twins or triplets, and $44.7 \%$ among singletons. Moreover, twins and triplets were independently associated with a higher rate of bottle-feeding: the odds ratio indicated that mothers who had twins or triplets were 2.44 times more likely to choose bottle-feeding with formula milk only than those who had singletons. Sucking ability at birth was associated with a higher rate of bottle-feeding: the odds ratio indicated that mothers who had infants with poor sucking ability at birth were 1.56 times more likely to choose bottle-feeding as those who had infants with normal sucking ability.

The documented benefits of breastfeeding compared with artificial feeding are numerous (Dewey et al., 1995; Goldman, 1993; Howie et al., 1990; Lucas et al., 1992). In particular, the importance of breast milk as the ideal nutrient for infants within the first 4 to 6 months after birth has been demonstrated. Breastfed infants have fewer respiratory tract infections and diarrheal illnesses (Howie et al., 1990). Increased mother-infant bonding and an overall decrease in infant morbidity and hospitalization rates have also been reported (Dewey et al., 1995; Forster et al., 1996). Further, one study suggested a beneficial effect of breast milk on infant's cognitive function (Lucas et al., 1992).
Meanwhile, a lot of twins and triplets are born as preterm infants with low birthweight (Arbuckle et al., 1993; Glinianaia et al., 2000; Kato, 2004). Infants who are born prematurely have greater need for the nutritional, anti-infective and immunologic advantages of breast milk. However, many preterm infants have poor sucking ability even when they are well, and it is reported that the rate of breastfeeding is low (Furman et al., 1998). Furthermore, it was indicated that even mothers of twins born at term were less likely to breastfeed compared to those of singleton births (Ford et al., 1994; Yokoyama \& Ooki, 2004).

There has been no population-based study on the rates of breastfeeding among singletons, twins and triplets in Japan. This present study was therefore carried out using population-based data to determine the rates of breastfeeding among singletons, twins and triplets in Japan, and identify factors associated with the decision to breastfeed or bottle-feed.

\section{Subjects and Methods}

In Japan, it has been established by the Maternal and Child Health Law that all infants should receive medical check-ups at less than 1 year, 1.5 years and 3 years of age. Pediatricians carry out the medical check-ups and public health nurses have a consultation on child-rearing at that time. Thus, these medical check-ups are given to almost $100 \%$ of children in Japan. The data from these medical check-ups are filed at the city public health department. These data, except personal information, are transferred into computerized files in Nishinomiya City. This city is a residential community and has a population of approximately 453,000. Birth numbers per year are about 4700 .

\section{Received 27 September, 2005; accepted 1 November, 2005.}

Address for correspondence: Yoshie Yokoyama, Faculty of Health Sciences, Okayama University Medical School, 2-5-1 Shikatacho, Okayama City, Okayama, 700-8558 Japan. E-mail: yyoko@md.okayama-u.ac.jp 
We analyzed the database of the medical check-ups for infants aged 3 to 6 months between April 2001 and July 2004. This database contains gestation number, gestational age, birthweight, parity, feeding methods at medical check-up, age of mothers, maternal health condition, maternal job and cooperation from other family members or relatives in childrearing. Information regarding feeding methods included breastfeeding only, mixed-feeding (mainly breast with some bottle or mainly bottle with some breast) and bottle-feeding with formula milk only. The definition used for exclusive breastfeeding was that the baby had been given breast milk only (Labbok \& Krasovec, 1991). Moreover, data on sucking ability at birth and use of an incubator were obtained from records in the Maternal and Child Health Handbook. This handbook was established by the Maternal and Child Health Law in Japan and is provided by the governor after a report of pregnancy. The purpose of this handbook is the maintenance of maternal and child's health and records: medical check-up during pregnancy and the condition of the newborn recorded by obstetricians, the progress of infant growth, periodic medical check-up for the infant and vaccinations recorded by pediatricians at medical check-ups.

With regard to statistical analyses, the independence of qualitative variables was examined using chi-square analysis. Analysis of variance (ANOVA) was conducted, with gestational age, birthweight and maternal age as dependent, and groups (singletons, twins and triplets; exclusive breastfeeding, mixedfeeding and bottle-feeding) as independent variables. Furthermore, logistic regression analysis was used to adjust for each factor associated with bottle-feeding. Variable in the models included gestation number, gestational age, parity, birthweight, incubator use, sucking ability at birth, infant age at medical examination, maternal health condition, maternal job, and cooperation from other family members or relatives for childrearing. The SPSS statistical package v11.5 for Windows (2002) was used for statistical analyses.

\section{$\overline{\text { Results }}$}

Between April 2001 and July 2004, 15,262 infants underwent the medical check-ups in Nishinomiya City. Overall, 14,963 $(98.0 \%)$ babies were singletons, $290(1.9 \%)$ were twins and $9(0.1 \%)$ were triplets. Exclusive breastfeeding was chosen by $6680(43.8 \%)$ mothers, mixed-feeding by 4645 (30.4\%) mothers, and bottle-feeding with formula milk only by 3900 $(25.6 \%)$ mothers. In $37(0.2 \%)$ mothers, feeding methods were unknown at the medical check-ups.

Table 1 summarizes the characteristics of the singletons, twins and triplets. There was a significant difference in the sucking ability at birth among the three groups: $1.0 \%$ of the singletons had poor sucking ability at birth, while the rate of that was $5.2 \%$ and $66.7 \%$ in twins and triplets, respectively. Moreover, a significant difference in incubator use was observed among the three groups: $9.4 \%$ of singletons were placed in an incubator at birth, while the rate was $49.7 \%$ and $100.0 \%$ in twins and triplets, respectively.

Table 2 shows infant factors associated with breastfeeding, mixed-feeding and bottle-feeding with formula milk only. Of the mothers who chose bottle-feeding, there was a significantly higher rate of mothers who had twins and/or triplets than those who chose exclusive breastfeeding or mixed-feeding $(p<.001)$. Infants of mothers who chose bottle-feeding had significantly lower gestational ages at birth than infants of those who chose exclusive breastfeeding or mixed-feeding ( $p$ $<.001)$. The birthweight of infants of mothers who chose bottle-feeding was significantly lower than that

\section{Table 1}

Major Characteristics of Subjects in the Singleton Group, Twin Group and Triplet Group

\begin{tabular}{|c|c|c|c|}
\hline & $\begin{array}{l}\text { Singleton } \\
n(\%)\end{array}$ & $\begin{array}{l}\text { Twin } \\
n(\%)\end{array}$ & $\begin{array}{c}\text { Triplet } \\
n(\%)\end{array}$ \\
\hline \multicolumn{4}{|c|}{ Gestational age (weeks) } \\
\hline$<32$ & $27(0.2)$ & $6(2.1)$ & $3(33.3)^{* * *}$ \\
\hline $32-35$ & $190(1.3)$ & $48(16.6)$ & $6(66.7)$ \\
\hline$\geq 36$ & $14,741(98.5)$ & $236(81.4)$ & $0(0.0)$ \\
\hline Mean $\pm S D$ & $39.0 \pm 1.42$ & $36.6 \pm 1.77$ & $32.0 \pm 1.73^{* * *}$ \\
\hline \multicolumn{4}{|l|}{ Birthweight } \\
\hline$\geq 2500 \mathrm{~g}$ & 13,994 (99.2) & $108(39.7)$ & $0(0.0)^{* * *}$ \\
\hline $1500 \mathrm{~g} \leq \mathrm{a}<2500 \mathrm{~g}$ & $739(5.0)$ & $154(56.6)$ & $5(62.5)$ \\
\hline$<1500 \mathrm{~g}$ & $25(0.2)$ & $10(3.7)$ & $3(37.5)$ \\
\hline Mean $\pm S D$ & $3035.7 \pm 387.9$ & $2553.1 \pm 423.8$ & $1745.5 \pm 404.9^{* * *}$ \\
\hline \multicolumn{4}{|l|}{ Parity } \\
\hline Primipara & $7888(52.7)$ & $176(60.7)$ & $9(100.0)^{* * *}$ \\
\hline Multipara & $7074(47.3)$ & $114(39.3)$ & $0(0.0)$ \\
\hline \multicolumn{4}{|l|}{ Incubator } \\
\hline Use & $1407(9.4)$ & $144(49.7)$ & $9(100.0)^{* * *}$ \\
\hline No use & $13,555(90.6)$ & $146(50.3)$ & $0(0.0)$ \\
\hline \multicolumn{4}{|l|}{ Sucking ability at birth } \\
\hline Normal & $14,818(99.0)$ & $275(94.8)$ & $3(33.3)^{* * *}$ \\
\hline Poor & $144(1.0)$ & $15(5.2)$ & $6(66.7)$ \\
\hline \multicolumn{4}{|l|}{ Maternal age (years) } \\
\hline Mean $\pm S D$ & $30.6 \pm 5.31$ & $31.4 \pm 4.21$ & $30.7 \pm 3.61 n s$ \\
\hline \multicolumn{4}{|c|}{ Maternal health condition } \\
\hline Good & $8361(55.9)$ & $126(43.4)$ & $3(33.3)^{* * *}$ \\
\hline Normal & $6186(41.3)$ & $155(53.4)$ & $6(66.7)$ \\
\hline Poor & $415(2.8)$ & $9(3.1)$ & $0(0.0)$ \\
\hline \multicolumn{4}{|l|}{ Maternal Job } \\
\hline No job & $12,849(85.9)$ & $254(87.6)$ & $9(100.0) n s$ \\
\hline Full-time job & $1725(11.5)$ & $34(11.7)$ & $0(0.0)$ \\
\hline Part-time job & $388(2.6)$ & $2(0.7)$ & $0(0.0)$ \\
\hline \multicolumn{4}{|c|}{$\begin{array}{l}\text { Cooperation from other family members } \\
\text { or relatives for childrearing }\end{array}$} \\
\hline Noncooperative & $3651(24.4)$ & $43(14.8)$ & $0(0.0)^{* * *}$ \\
\hline Cooperative & $11,311(75.6)$ & 247 (85.2) & $9(100.0)$ \\
\hline
\end{tabular}

Note: ${ }^{* *} p<.001, n s=$ no significance. 
Table 2

Infant Factors Associated With Breastfeeding, Mixed-Feeding and Bottle-Feeding

\begin{tabular}{|c|c|c|c|}
\hline & $\begin{array}{c}\text { Exclusive } \\
\text { breastfeeding } \\
n(\%)\end{array}$ & $\begin{array}{c}\text { Mixed-feeding } \\
n(\%)\end{array}$ & $\begin{array}{c}\text { Bottle-feeding } \\
n(\%)\end{array}$ \\
\hline \multicolumn{4}{|l|}{ Gestation number } \\
\hline Singleton & $6668(44.7)$ & $4516(30.3)$ & $3744(25.0)^{* * *}$ \\
\hline Twin or triplet & $12(4.1)$ & $129(43.4)$ & $156(52.5)$ \\
\hline \multicolumn{4}{|c|}{ Gestational age (weeks) } \\
\hline$<32$ & $9(25.0)$ & $9(25.0)$ & $18(50.0)^{* * *}$ \\
\hline $32-35$ & $50(20.5)$ & $77(31.6)$ & $117(48.0)$ \\
\hline$\geq 36$ & $6620(44.3)$ & $4559(30.5)$ & $3762(25.2)$ \\
\hline Mean $\pm S D$ & $39.1 \pm 1.33$ & $39.0 \pm 1.46$ & $38.8 \pm 1.68^{* * *}$ \\
\hline \multicolumn{4}{|l|}{ Parity } \\
\hline Primipara & $3293(40.9)$ & $2716(33.7)$ & $2042(25.4)^{* * *}$ \\
\hline Multipara & $3387(47.2)$ & $1929(26.9)$ & $1858(25.9)$ \\
\hline \multicolumn{4}{|l|}{ Birthweight } \\
\hline$\geq 2500 \mathrm{~g}$ & $6345(45.1)$ & $4247(30.2)$ & $3477(24.7)^{* * *}$ \\
\hline $1500 \mathrm{~g} \leq \mathrm{a}<2500 \mathrm{~g}$ & $325(29.3)$ & $386(34.8)$ & $398(35.9)$ \\
\hline$<1500 \mathrm{~g}$ & $10(21.3)$ & $12(25.5)$ & $25(53.2)$ \\
\hline Mean $\pm S D$ & $3074.6 \pm 372.8$ & $3031.7 \pm 412.9$ & $3002.2 \pm 450.0^{* * *}$ \\
\hline \multicolumn{4}{|l|}{ Incubator } \\
\hline No use & $6159(45.1)$ & $4160(30.4)$ & $3352(24.5)^{* * *}$ \\
\hline Use & $521(33.5)$ & $485(31.2)$ & $548(35.3)$ \\
\hline \multicolumn{4}{|l|}{ Sucking ability at birth } \\
\hline Normal & $6644(44.1)$ & $4590(30.5)$ & $3826(25.4)^{* * *}$ \\
\hline Poor & $36(21.8)$ & $55(33.4)$ & $74(44.8)$ \\
\hline \multicolumn{4}{|c|}{ Infant age at medical examination } \\
\hline 3 months & $1672(43.8)$ & $1238(32.5)$ & $903(23.7)^{* * *}$ \\
\hline 4 months & $4859(44.4)$ & $3286(30.0)$ & $2796(25.6)$ \\
\hline 5 months & $128(31.8)$ & $105(26.2)$ & $169(42.0)$ \\
\hline 6 months & $21(30.4)$ & $16(23.2)$ & $32(46.4)$ \\
\hline Mean $\pm S D$ & $3.78 \pm 0.48$ & $3.76 \pm 0.50$ & $3.83 \pm 0.53$ \\
\hline
\end{tabular}

of infants of mothers who chose exclusive breastfeeding or mixed-feeding $(p<.001)$. From the mothers who chose bottle-feeding, there was a significantly higher rate of infants who were placed in an incubator at birth than from those who chose exclusive breastfeeding or mixed-feeding $(p<.001)$. Moreover, the age of infants at the medical check-up of mothers who chose bottle-feeding was significantly older than that of mothers who chose exclusive breastfeeding or mixed-feeding $(p<.001)$.

Table 3 shows maternal factors associated with breastfeeding, mixed-feeding and bottle-feeding with formula milk only. The average age of mothers who chose bottle-feeding was significantly younger than those who chose exclusive breastfeeding or mixedfeeding $(p<.001)$. There was a significantly higher rate of mothers in poor health from the mothers who chose bottle-feeding than from those who chose exclusive breastfeeding or mixed-feeding $(p<.001)$.
Table 3

Maternal Factors Associated With Breastfeeding, Mixed-Feeding and Bottle-Feeding

\begin{tabular}{|c|c|c|c|}
\hline & $\begin{array}{c}\text { Exclusive } \\
\text { breastfeeding } \\
n(\%)\end{array}$ & $\begin{array}{c}\text { Mixed-feeding } \\
n(\%)\end{array}$ & $\begin{array}{c}\text { Bottle-feeding } \\
n(\%)\end{array}$ \\
\hline \multicolumn{4}{|l|}{ Maternal age } \\
\hline$<20$ & $18(18.8)$ & $14(14.6)$ & $64(66.7)^{* * *}$ \\
\hline $20-29$ & $2496(43.6)$ & $1555(27.2)$ & $1673(29.2)$ \\
\hline $30-39$ & $4058(44.7)$ & $2955(32.5)$ & $2075(22.8)$ \\
\hline$\geq 40$ & $84(33.1)$ & $102(40.2)$ & $68(26.8)$ \\
\hline Mean $\pm S D$ & $30.7 \pm 3.81$ & $31.1 \pm 4.03$ & $30.1 \pm 8.04 * * *$ \\
\hline \multicolumn{4}{|c|}{ Maternal health condition } \\
\hline Good & $3963(46.8)$ & $2433(28.7)$ & $2075(24.5)^{* * *}$ \\
\hline Normal & $2565(40.5)$ & $2074(32.8)$ & $1692(26.7)$ \\
\hline Poor & $152(36.0)$ & $138(32.6)$ & $133(31.4)$ \\
\hline \multicolumn{4}{|l|}{ Maternal Job } \\
\hline No job & $5780(44.2)$ & 3918 (29.9) & $3384(25.9)^{* * *}$ \\
\hline $\begin{array}{l}\text { Full-time job } \\
\text { or part-time job }\end{array}$ & $900(42.0)$ & $727(33.9)$ & $516(24.1)$ \\
\hline \multicolumn{4}{|c|}{$\begin{array}{l}\text { Cooperation from other family members } \\
\text { or relatives for childrearing }\end{array}$} \\
\hline Noncooperative & $1684(45.7)$ & $1095(29.7)$ & $903(24.5)^{*}$ \\
\hline Cooperative & 4996 (43.2) & $3550(30.8)$ & $2997(26.0)$ \\
\hline
\end{tabular}

Table 4 shows the results of the logistic regression analysis of bottle-feeding with associated factors as independent variables. Twins and triplets were independently associated with a higher rate of bottle-feeding: the odds ratio indicated that mothers who had twins and triplets were 2.44 times more

\section{Table 4}

Results of Logistic Regression on Bottle-feeding With Formula Milk Only and Associated Factors

\begin{tabular}{llc}
\hline Independent variables & Odds ratio & 95\% confidence interval \\
\hline $\begin{array}{l}\text { Gestation number } \\
\quad \text { Singleton }\end{array}$ & 1.00 & \\
$\quad$ Twins and Triplets & $2.44^{* * *}$ & $1.90-3.13$ \\
Incubator & & \\
$\quad$ No use & 1.00 & $1.19-1.53$ \\
$\quad$ Use & $1.35^{* *}$ & \\
Sucking ability at birth & & \\
$\quad$ Normal & 1.00 & $1.12-2.18$ \\
$\quad$ Poor & $1.56 * *$ & \\
Infant age at medical & & \\
examination & & $1.43-2.15$ \\
3-4 months & 1.00 & \\
5-6 months & $1.75^{* * *}$ & $1.08-1.66$ \\
Maternal health condition & & \\
Good-normal & 1.00 & \\
Poor & $1.34^{* *}$ & \\
\hline
\end{tabular}

Note: ${ }^{* *} p<.01,{ }^{* * *} p<.001$. 
likely to choose bottle-feeding with formula milk only than those who had singletons. The age of infants was associated with a higher rate of bottle-feeding: the odds ratio indicated that mothers who had infants aged 5 to 6 months were 1.78 times more likely to choose bottle-feeding formula milk only as those who had infants aged 3 to 4 months. Sucking ability at birth was associated with a higher rate of bottlefeeding: the odds ratio indicated that mothers who had infants with poor sucking ability at birth were 1.56 times more likely to choose bottle-feeding formula milk only than those who had infants with normal sucking ability. Furthermore, use of an incubator and maternal poor health were associated with a higher rate of bottle-feeding. Bottle-feeding was not associated with gestation number, gestational age, parity, birthweight, incubator, sucking ability at birth, infant age at medical examination, maternal health condition, maternal job, and cooperation from other family members or relatives for childrearing

\section{Discussion}

In our population-based study, the rate of exclusive breastfeeding among twins or triplets aged 3 to 6 months was considerably lower than that of singleton babies aged 3 to 6 months: $4.1 \%$ among twins or triplets, and $44.7 \%$ among singletons. The rate of exclusive breastfeeding varies according to each country (Antoniou et al., 2005; Czeszyñska \& Kowalik, 1998; Ford et al., 1995; Heck et al., 2003; Leung et al., 2002). Since a program of breastfeeding promotion was performed in Poland, the rate of exclusive breastfeeding in the Polish singleton population (73.2\% of infants; Czeszyñska \& Kowalik, 1998) has exceeded that reported by the present study. However, exclusive breastfeeding among multiple babies was observed very rarely and the rate reported in Poland (4.9\%; Czeszyñska \& Kowalik, 1998) was similar to that of the present study.

Ford et al. (1994) indicated that twin pregnancy was a factor adversely affecting the initiation of breastfeeding. In addition, results of logistic regression in this study showed that mothers with twins or triplets were twice as likely to choose bottle-feeding with formula milk only than mothers with singletons, and choice of bottle-feeding was not associated with low birthweight. These results are consistent with those reported by Ford et al. (1994). Altogether, these results suggest that establishment of breastfeeding after multiple births is extremely difficult.

Meanwhile, it was reported that there was no significant difference in the success of breastfeeding between singleton and twin infants, if there was a program to establish breastfeeding and support provided by experienced staff (Liang et al., 1997). In addition, Leonard (2003) indicated that families with multiple birth infants required assistance from healthcare providers who were encouraging, knowledgeable and skilled in the techniques of breastfeeding multiple birth children. A program to establish breastfeeding for multiple births should be developed and healthcare providers for families with multiple births are necessary in Japan.

Although this study is not a longitudinal study, the rate of exclusive breastfeeding declined steadily according to the age of infants, from $43.8 \%$ in infants aged 3 months (12-15 weeks) to $30.4 \%$ in infants aged 6 months (24-27 weeks). These are lower compared with the study of Ford et al. (1995) in New Zealand, who found a progressive fall after birth in the rate of exclusive breastfeeding, from $68 \%$ at 8 to 12 weeks to $36 \%$ by 19 to 24 weeks.

Concerning factors associated with the decision to breastfeed or bottle-feed, this study revealed that sucking ability at birth was a significant factor. Mothers who had infants with poor sucking ability were more likely to choose bottle-feeding with formula milk only. As infants with poor sucking ability at birth often need nasogastric tube feeding or intravenous nutrition, the establishment of breastfeeding for such infants is more difficult than for infants with normal sucking ability. Accordingly, mothers who have infants with poor sucking ability should be given support in establishing and maintaining lactation, including education on how to milk regularly and store it in sanitary conditions.

Mezzacappa et al. (2002) found that the frequency of bottle-feeding was positively associated with perceived maternal stress. In this study, mothers in poor health were more likely to choose bottle-feeding with formula milk only. This result is consistent with that reported by Mezzacappa et al. (2002). While several studies (Ford et al., 1994; Piper \& Parks, 1996) provided evidence that mothers of singletons who breastfed were older, maternal age at delivery was not a factor associated with the decision to breastfeed or bottle-feed for multiple births.

Data on cigarette smoking of mothers, quantity of maternal lactation and the use of a dummy were lacking in this study, and it has been suggested that these factors are associated with the initiation and duration of breastfeeding (Furman et al., 1998; Victora et al., 1993). Other studies (Furman et al., 1998; Liang et al., 1997) have attempted to assess the rate of breastfeeding at discharge. These factors and other aspects will be investigated in the future.

\section{Acknowledgment}

This research was supported by Ministry of Education, Science, Sports and Culture, Grant-in-Aid for Scientific Research (B), 2004-2007.

\section{References}

Antoniou, E., Daglas, M., Iatrakis, G., Kourounis, G., \& Greatsas, G. (2005). Factors associated with initiation and duration of breastfeeding in Greece. Clinical and Experimental Obstetrics and Gynecology, 32, 37-40. 
Arbuckle, T. E., Wilkins, R., \& Sherman, G. J. (1993). Birth weight percentiles by gestational age in Canada. Obstetrics and Gynecology, 81, 39-48.

Czeszyñska, M. B., \& Kowalik, K. (1998). Multiple pregnancy: Factors contributing to early infant's breast-feeding - Own experience. Acta Geneticae Medicae et Gemellologiae, 47, 191-196.

Dewey, K. G., Heining, M. J., \& Nommsen-Rivers, L. A. (1995). Differences in morbidity between breast-fed and formula fed infants. The Journal of Pediatrics, 126, 696-702.

Ford, R. P. K., Mitchell, E. A., Scragg, R., Stewart, A. W., Taylor, B. J., \& Allen, E. M. (1994). Factors adversely associated with breast feeding in New Zealand. Journal of Paediatrics and Child Health, 30, 483-489.

Ford, R. P. K., Wild, C. J., Mitchell, E. A., \& Tuohy, P. (1995). Infant feeding patterns in Canterbury. New Zealand Medical Journal, 108, 59-61.

Forster, S. F., Slade, P., \& Wilson, K. (1996). Body image, maternal fetal attachment, and breast feeding. Journal of Psychosomatic Research, 41, 181-184.

Furman, L., Minich, N. M., \& Hack, M. (1998). Breastfeeding of very low birth weight infants. Journal of Human Lactation, 14, 29-34.

Glinianaia, S. V., Skjaerven, R., \& Magnus, P. (2000). Birthweight percentiles by gestational age in multiple births. A population-based study of Norwegian twins and triplets. Acta Obstetricia et Gynecologica Scandinavica, 79, 450-458.

Goldman, A. S. (1993). The immune system of human milk. Antimicrobial, anti-inflammatory and immunodulative properties. The Pediatric Infectious Disease Journal, 12, 664-672.

Heck, K. E., Schoendorf, K. C., Chavez, G. F., \& Braveman, P. (2003). Does postpartum length of stay affect breastfeeding duration? A population-based study. Birth, 30, 153-159.
Howie, R. W., Forsyth, J. S., Ogston, S. A., Clark, A., \& Florey, C. (1990). Protective effect of breast-feeding against infection. British Medical Journal, 300, 11-16.

Kato, N. (2004). Reference birthweight range for multiple birth neonates in Japan. BMC Pregnancy and childbirth, 4, 1-9.

Labbok, M., \& Krasovec, K. (1991). Towards consistency in breastfeeding definitions. Studies in Family Planning, 21, 226-230.

Leung, G. M., Ho, L. M., \& Lam, T. H. (2002). Breastfeeding rates in Hong Kong: A comparison of the 1987 and 1997 birth cohorts. Birth, 29, 162-168.

Liang, R., Gunn, A. J., \& Gunn, T. R. (1997). Can preterm twins breast feed successfully? New Zealand Medical Journal, 13, 209-212.

Leonard, L. G. (2003). Breastfeeding rights of multiple birth families and guidelines for health professionals. Twin Research, 6, 34-45.

Lucas, A., Morley, R., Cole, T. J., Lister, G., \& LeesonPayne, C. (1992). Breast milk and subsequent intelligence quotient in children born preterm. Lancet, 339, 261-64.

Mezzacappa, E. S., Guethlein, W., \& Katkin, E. S. (2002). Breast-feeding and maternal health in online mothers. Annals of Behavioral Medicine, 24, 299-309.

Piper, S., \& Parks P. L. (1996). Predicting the duration of lactation: Evidence from a national survey. Birth, 23, 7-12.

SPSS Inc. (2002). SPSS for Windows (Release 11.5) [Computer software]. Chicago, IL: SPSS Inc.

Victora, C. G., Tomasi, E., Olinto, M. T., \& Barros, F. C. (1993). Use of pacifiers and breastfeeding duration. Lancet, 341, 404-406.

Yokoyama, Y., \& Ooki, S. (2004). Breast-feeding and bottle-feeding of twins, triplets and higher order multiple births. Japanese Journal of Public Health, 51, 969-974. 